\title{
Comment on: International Hernia Collaboration Consensus on Nomenclature of Abdominal Wall Hernia Repair: Reply
}

\author{
Samuel G. Parker ${ }^{1} \cdot$ Christopher P. J. Wood $^{1} \cdot$ David L. Sanders $^{2}$ • \\ Alastair C. J. Windsor ${ }^{1}$
}

Published online: 6 September 2017

(C) Société Internationale de Chirurgie 2017

\section{Dear Sir,}

We read with interest the International Hernia Collaboration's (IHCs) article on abdominal wall nomenclature. This article reports a survey that was carried out using the IHCs Facebook group. Over 100 hernia experts completed the survey which aimed to define the terms used to describe the anatomical planes of the abdominal wall. The results show a preference for the 'anatomical' terms (retro-muscular or retro-rectus, pre-peritoneal and intra-peritoneal) as opposed to the 'colloquial' terms (sublay, underlay and IPOM) in the three deepest abdominal wall planes. Going forwards, these anatomical terms should be popularised for standardisation of the nomenclature and to prevent nomenclature misuse and confusion.

Academic hernia surgeons must, however, be aware of the past failings in the literature. As the terms have been used inconsistently in the past, academics performing study comparisons or meta-analysis in the future, should read the anatomical descriptions of the abdominal wall plane used (for mesh insertion) and not simply assume that the authors used these terms correctly.
The survey shows no definitive result for the abdominal wall plane in position C. It suggests that both the terms 'retro-muscular' and 'retro-rectus' should be used interchangeably. We suggest that the term 'retro-rectus' is more anatomically correct when no TAR has been performed and the mesh is inserted into the retro-rectus pocket. The term 'retro-muscular' should be used after a TAR has been performed where the plane extends laterally and is posterior to the abdominal strap muscles (external oblique, internal oblique and transversalis fascia).

We accept the results of the IHC survey. Together with the authors of the IHC article, we should look into ways of publicising and propagating this terminology so that future publications use this standardised nomenclature and the inconsistencies cease to exist.

Compliance with ethical standards

Conflict of interest All authors declare that they have no conflict of interest.
Samuel G. Parker

samgparker@nhs.net

1 Department of General and Colorectal Surgery, University College Hospital London, 235 Euston Road, London NW1 2BU, UK

2 Department of General and Upper GI Surgery, North Devon District Hospital, Raleigh Park, Barnstaple, Devon EX31 4JB, UK 